\title{
Anomalous Hall effect and chiral spin liquid in a metallic pyrochlore
}

Time-reversal symmetry breaking and spontaneous Hall effect without magnetic order

Authors: Yo Machida, Saturo Nakatsuji, Shigeki Onoda, Takashi Tayama, and Toshiro Sakakibara

Nature, 463 210, 2010.

\section{Recommendation and Commentary by Leon Balents, KITP, UCSB}

The subject of this recommendation, $\mathrm{Pr}_{2} \mathrm{Ti}_{2} \mathrm{O}_{7}$, represents an intriguing confluence of two streams of research. Pyrochlores, with the chemical formula $\mathrm{A}_{2} \mathrm{~B}_{2} \mathrm{X}_{7}$, form a cubic structure consisting of two interpenetrating networks of corner-sharing tetrahedra of $\mathrm{A}$ and $\mathrm{B}$ atoms. Highly insulating rare earth pyrochlores, where $\mathrm{A}$ is a lanthanide element and $\mathrm{B}$ is nonmagnetic (especially $\mathrm{B}=\mathrm{Ti}$ ), have long been studied in the magnetism community. In recent years, attention has focused on $\mathrm{Dy}_{2} \mathrm{Ti}_{2} \mathrm{O}_{7}$ and $\mathrm{Ho}_{2} \mathrm{Ti}_{2} \mathrm{O}_{7}$, which host "spin ice" physics, with localized $4 \mathrm{f}$ electrons forming large classical spins that enjoy interesting fluctuation physics due to the high degree of frustration of the pyrochlore lattice (see the previous journal club entry here: http://www.condmatjournalclub.org/?p=799). Independently, researchers have been studying unusual metal-insulator transitions in iridium pyrochlores, $\mathrm{A}_{2} \mathrm{Ir}_{2} \mathrm{O}_{7}$, where $\mathrm{A}$ is again a lanthanide. Here the focus is on conducting $5 \mathrm{~d}$ electrons on the $\mathrm{Ir}^{4+}$ ions, which are interesting because of the combination of strong spin-orbit coupling and correlations. In most of the iridates, the $4 \mathrm{f}$ spins are bystanders, just reacting to the Mott metal-insulator transition driven by the d electrons.

Uniquely among this series of materials, $\operatorname{Pr}_{2} \mathrm{Ir}_{2} \mathrm{O}_{7}$ remains metallic at all temperatures, avoiding magnetic ordering of the $\mathrm{Ir}^{4+}$ ions which accompanies the Mott transition, and which appears to enslave the rare earth moments in the other cases. This allows non-trivial spin physics of the rare earth Pr moments to reappear. The combination of frustrated magnetism of local Pr spins coupled to the moderately correlated and spin-orbit coupled Ir conduction electrons make this an extremely complex and rich system!

The physics discussed in the paper highlighted above is associated with the coupling of the spins to the local moments. This is known to be substantial, and results in dramatic signals of the magnetism in the Hall conductivity, 
known as the Anomalous Hall Effect (AHE). Typically the AHE is observed in ferromagnets, where it is proportional to the sample magnetization. It has been suggested theoretically, however, that an anomalous Hall effect can arise not from a uniform magnetization but rather a non-coplanar spin texture, characterized by the scalar spin chirality, $\vec{S}_{i} \cdot \vec{S}_{j} \times \vec{S}_{k}$, which quantifies the degree of non-coplanarity of three nearby spins $(\mathrm{i}, \mathrm{j}, \mathrm{k})$. A physical explanation of this effect is that as a moving electron's spin follows that of the local moments $\vec{S}_{i}$, it acquires a Berry phase proportional to the solid angle subtended by the three vectors. This Berry phase acts similarly to the phase accumulated by motion in a magnetic field, likewise producing a Hall effect. In prior work on $\mathrm{Pr}_{2} \mathrm{Ir}_{2} \mathrm{O}_{7}[1]$, a large AHE was observed in applied fields, and shown not to track the sample magnetization but instead correlate with the scalar spin chirality. Non-coplanar spin configurations are indeed expected due to the non-collinear local Ising axes at different Pr sites, and the fieldinduced AHE was shown to be in accord with the expectations based on such anisotropy.

What about zero field? The experiments in the highlighted paper present an intriguing answer to this question. They find that the AHE is present even in zero field. This in itself is not surprising if the spins freeze or order into a non-coplanar configuration with non-vanishing average spin chirality. However, while spin freezing (and a small hysteretic magnetization) is observed experimentally below $T_{f} \approx 300 \mathrm{mK}$, a zero field AHE is observed already once the sample is cooled to $1.5 \mathrm{~K}$. A non-zero Hall conductivity is a direct indication of broken time-reversal symmetry. What is this intermediate state with broken time-reversal symmetry but no spin freezing? The authors suggest it should be regarded as a chiral spin liquid.

A chiral spin liquid state, in which no static moment is present, $\left\langle\vec{S}_{i}\right\rangle=0$, but scalar spin chirality is non-zero, was proposed by Kalmeyer and Laughlin in 1987 as a realization of Anderson's quantum spin liquid of Heisenberg spins on the triangular lattice, stabilized by quantum fluctuations. The Kalmeyer-Laughlin state not only breaks time-reversal symmetry, but hosts exotic quasiparticles, spinons, with fractional spin and statistics. Might similarly exotic physics be behind intermediate temperature AHE in $\operatorname{Pr}_{2} \mathrm{Ir}_{2} \mathrm{O}_{7}$ ?

One may want to be cautious. The putative chiral spin liquid does not extend to zero temperature (static moments are present below $T_{f}$ ), so that the spin fluctuations involved might be predominantly classical thermal ones rather than quantum. Indeed, this range of temperatures is similar to the one in which the spins in spin ice, which are almost perfectly classical, remain 
dynamic though strongly correlated. Yet even if the explanation is in part classical, a chiral spin liquid could be a distinct phase of matter (it would definitely be in a system with spin-rotation symmetry), and certainly has never been observed prior to this.

Many interesting questions remain. What is the role of the strong spin orbit interactions operative for both the Pr spins and the Ir electrons? Does proximity to the Mott transition seen in other pyrochlore iridates have any significant implications? How far can the analogy to spin ice be pushed? If it is good, can the magnetic monopoles identified in spin ice be somehow measured by AHE? Might quantum fluctuations of the Pr spins play a role? The $\mathrm{Pr}^{3+}$ ion has a much reduced dipole moment compared to the classical spin ices, and is a non-Kramer's ion: these properties tend to reduce the classicality of the spins and may encourage exotic multipolar magnetic correlations. Numerous qualitative and quantitative experimental facts have not been fully explained, including logarithmic growth of the susceptibility and $T^{1 / 2}$ dependence of the specific heat at low temperature, and indications of Kondo effect and two Curie-Weiss regimes. The richness of $\mathrm{Pr}_{2} \mathrm{Ir}_{2} \mathrm{O}_{7}$ provides both stimulation and challenges for much future study.

\section{References}

[1] Y. Machida, S. Nakatsuji, Y. Maeno, T. Tayama, T. Sakakibara, and S. Onoda. Unconventional Anomalous Hall Effect Enhanced by a Noncoplanar Spin Texture in the Frustrated Kondo Lattice $\operatorname{Pr}_{2} \mathrm{Ir}_{2} \mathrm{O}_{7}$. Phys. Rev. Lett., 98:057203, Jan 2007. 